\section{Lick the nasty habit}

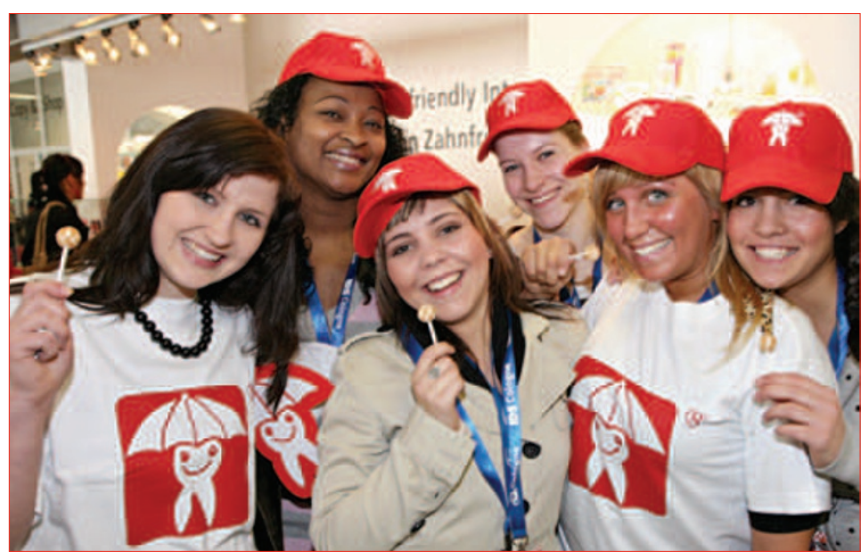

Dental professionals have a tempting new alternative to try and persuade patients to quit smoking: sugar-free lollipops may help to overcome the oral fixation. After quitting, many smokers search for alternative products to help satisfy the hand-to-mouth fixation of cigarettes. Lollipops are often preferred because they offer a cigarette-like sucking sensation.

Millions of smokers visit a dentist every year for a dental checkup. Members of the dental team can therefore play a major role in helping people give up smoking. While it is recommended to advise patients to swap their cigarettes for lollipops, be sure to endorse a toothfriendly alternative.

Toothfriendly International is supporting quitters with their sugarfree lollipops. To claim your free toothfriendly lollipop kit, send your name and delivery address to contact@toothfriendly.ch.

\section{Precision handling}

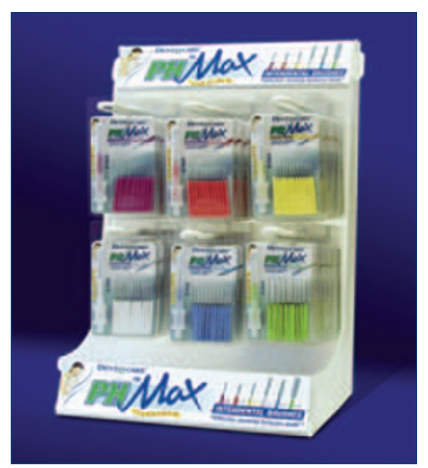

Dent-O-Care has launched its new flexible $\mathrm{PH}$ Max range. $\mathrm{PH}$ Max means Precision Handling with Maximum benefit and each pack has 12 brushes, a grip holder and an extension handle that doubles as a protective cap.

Unlike other hand-held designs, there is no need to reshape the brush before using. Flexing the handle with the index finger tilts the brush head to achieve the best angle for cleaning between teeth. For maximum control, attach both extensions before flexing the original handle.

The range of six sizes with plastic coated cores $(0.4-0.9 \mathrm{~mm})$ is also

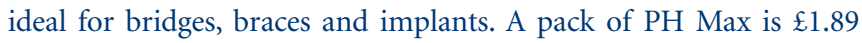
with a suggested patient price of $\mathfrak{E 3 . 4 9}$. Patient test packs containing one of each size plus attachments are $80 \mathrm{p}$. The company is currently offering the $\mathrm{PH}$ Max unit worth $\mathfrak{£} 15$ plus one dozen patient test packs free, when you order 72 packs of PH Max from Dent-O-Care, until 30 June 2007.

For the offer, a free sample pack and further information, telephone free on 08009801517 or email kproven@dentocare.co.uk.

\section{Easy to swallow seaweed}

Molar Ltd has announced that ProDen PlaqueOff is now being produced in a new easy to swallow capsule format. ProDen PlaqueOff is particularly useful for those patients who have problems controlling plaque and calculus using traditional methods.

By taking two capsules daily, reductions in calculus, plaque and bad breath are normally seen

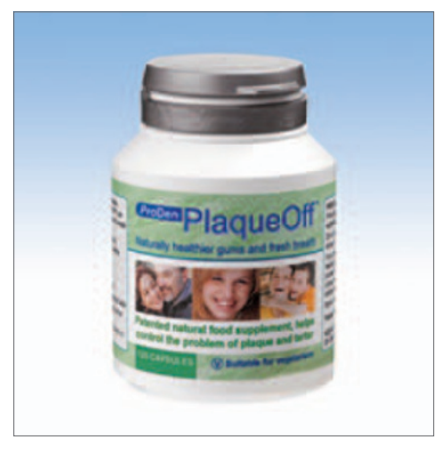
within five to eight weeks. The patented formulation contains only specially selected seaweed from Norway and Iceland. The new capsules are suitable for vegetarians, easy to swallow and have an improved taste.

The new capsule format will be available to order from your regular wholesaler (new stock will be phased in as old stock is depleted). Telephone 01934710022 or email info@molarltd.co.uk for more information.

\section{Fully ambidextrous}

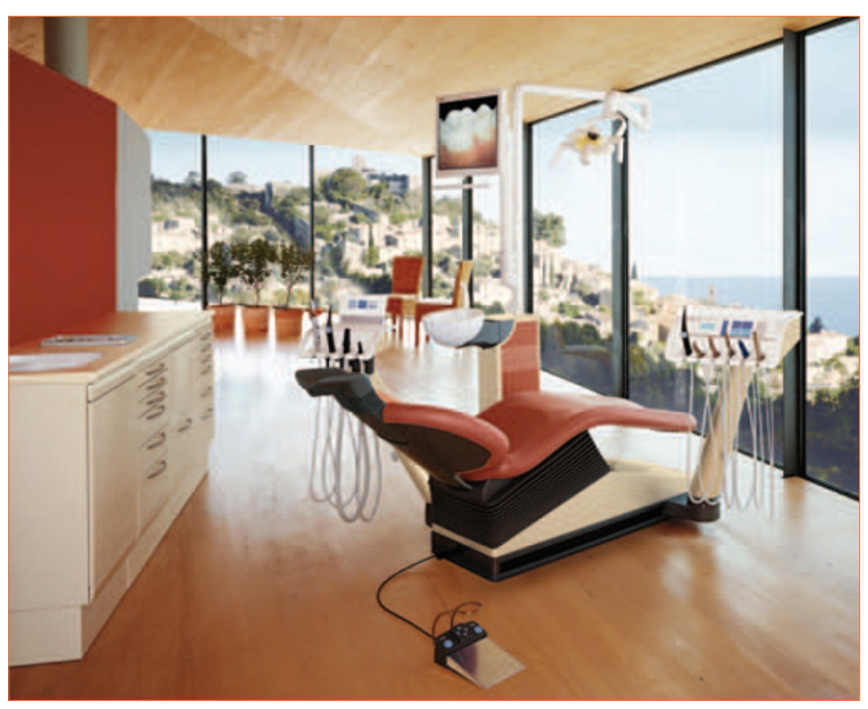

The Pelton and Crane Ellipse Dental unit from Henry Schein provides versatility with comfort. The Ellipse accommodates left or right handed dentists with equal facility as it is fully ambidextrous, the chair also swivels on its axis allowing for perfect specialist treatment, and all chair controls are easily accessible by the operator or the nurse.

The ErgoSoothe fully integrated lumbar and back massaging system, in association with the soft and ultra durable Ultraleather upholstery, offers the patient total comfort. The operating light features a feathered-edge pattern to reduce eye fatigue and combines with a triple axis operating light head to allow a wider range of working positions.

The quartz halogen lamp is easily replaced when required via a hinged light back. The Pelton and Crane units offer full flexibility, yet integrating all features required in the modern dental practice. Call 08700102041 for further information. 